\title{
Inhibition of the p38 MAPK pathway sensitises human colon cancer cells to 5-fluorouracil treatment
}

\author{
SHI YU YANG ${ }^{1}$, ANUR MIAH ${ }^{1}$, KEVIN M. SALES ${ }^{1}$, BARRY FULLER $^{1}$, \\ ALEXANDER M. SEIFALIAN ${ }^{1}$ and MARC WINSLET ${ }^{1,2,3}$ \\ ${ }^{1}$ Division of Surgery and Interventional Science, University College London, Rowland Hill Street, London; \\ ${ }^{2}$ Royal Free Hampstead NHS Trust Hospital; ${ }^{3}$ University College Hospital, London, UK
}

Received November 29, 2010; Accepted January 10, 2011

DOI: $10.3892 /$ ijo.2011.982

\begin{abstract}
Colorectal cancer is the third most common cause of cancer-related deaths in the Western world. 5-Fluorouracil (5-FU) based chemotherapeutic regimes have been the mainstay of systemic treatment for disseminated colorectal cancer for many years. However, it only produces a $25 \%$ response rate due to the drug-resistance. The mitogen-activated protein kinase (MAPK) pathway is involved in the anti-apoptotic process; its activation provides cancer cells with a survival advantage to escape the apoptotic challenge. This study assessed whether the p38 MAPK pathway is involved in 5-FU resistance in colorectal cancer cells. 5-FU only or 5-FU combined with a p38 MAPK pathway inhibitor (SB203580) was used to treat 5-FU-resistant colorectal cancer cells. The effect of the treatment on cell viability, death and caspase activities was assessed. Western blotting was used to investigate the responses of apoptosis-related proteins following the treatment. Results showed that p38 MAPK inhibitor significantly increased colorectal cancer cell sensitivity to 5-FU. SB203580 in combination with 5 -FU significantly reduced cell viability $(\mathrm{P}<0.01)$, and increased cell death and cellular caspase activity $(\mathrm{P}<0.01)$. Western blotting data revealed that SB203580 sensitises cancer cells to 5-FU due to an increase in Bax expression. These findings suggest that p38 MAPK is involved in cancer cell survival, and that the inhibition of p38 MAPK can enhance 5-FU to kill colorectal cancer cells.
\end{abstract}

\section{Introduction}

Colorectal cancer (CRC) is the third most common cause of cancer-related deaths among men and women in the Western

Correspondence to: Dr Shi Yu Yang, Division of Surgery and Interventional Science, UCL Medical School, University College London, Royal Free Campus, Rowland Hill Street, London NW3 2PF, UK

E-mail: shiyu.yang@medsch.ucl.ac.uk

Key words: colorectal cancer, p38 mitogen-activated protein kinase, fluorouracil, chemoresistance world $(1,2)$. Europe has the highest CRC incidence with an estimated 412,900 new cases in 2006 (1) closely followed by the USA which had 145,290 colorectal cancers in 2008 (3). 5-Fluorouracil (5-FU)-based chemotherapeutic regimes have been the mainstay of systemic treatments in CRC for many years. However 5-FU treatment is not very effective against disseminated CRC, overall it only produces $10-20 \%$ response rate (4). One major reason for 5-FU failure to treat disseminated $\mathrm{CRC}$ is the development of drug resistance (5). Tumours usually consist of mixed populations of malignant cells, some of which are drug-sensitive while others are drug-resistant. Chemotherapy kills drug-sensitive cells but leaves behind a higher proportion of drug-resistant cells, which can expand and maintain tumour growth in spite of repeated treatment. One way to overcome drug-resistance is to sensitise the cancer cells to 5-FU treatment.

5 -FU is an anti-tumour drug widely used in the treatment of solid tumours (6). Its anti-tumour mechanism has been associated with inhibition of thymidylate synthase (TS) and induction of genotoxic stress by incorporation of 5-FU into RNA and DNA (6). Resistance to 5-FU is a multi-factorial event which includes altered drug-transport mechanisms, metabolic conversion, molecular mechanisms, protection from apoptosis and resistance via cell cycle kinetics (7). Because of the reactivity of 5-FU and complexity of the cellular response to DNA and RNA damage, 5-FU-induced apoptotic signalling is likely to involve several pathways. Elucidation of the details of these pathways will help us to understand why some tumour cells exposed to 5-FU often lose sensitivity and become resistant to apoptotic signals.

Chemotherapy-reduced genotoxic stress often induces multiple signal transduction pathways including mitogen-activation protein kinase (MAPK). MAPK has been associated with anti-apoptotic processes and chemo-resistance development in CRC cells (8). MAPK has been found to be activated aberrantly in CRC patients (9). We hypothesised that MAPK activation may provide cancer cells with a survival advantage, and assist malignant cells escaping from the drug-induced apoptotic challenge. To test this, we treated 5-FU-resistant CRC cells with 5-FU supplemented with or without MAPK pathway inhibitors, and assessed the effects of the treatment on cell viability, cell death and caspase activity. It was found that inhibition of p38 MAPK pathway increased colorectal 
cancer cell (HT29) sensitivity to 5-FU treatment. Further studies showed that the increased sensitivity to 5-FU treatment was mediated with the elevation of a pro-apoptotic protein-Bax.

\section{Material and methods}

Reagents. Caspase 3/7, 8 and 9 assay kits and cell viability assay kits were purchased from Promega. Yo Pro-1 iodide was purchased from Invitrogen. p38 MAPK and MEK1/2 MAPK inhibitors were purchased from Merck Bioscience. Antibodies against human p38 and phospho-p38, p42/44 and phosphor-p42/44 were purchased from New England Bio-labs. Anti-human Bax and Bcl2 antibodies were purchased from Santa Cruz Biotechnology. 5-Fluorouracil $(50 \mathrm{mg} / \mathrm{ml})$ was manufactured in Medac (Germany) and purchased from the Royal Free Hospital pharmacy department. All cell culture media, sera and antibiotics were purchased from Gibco.

Cell lines and culture condition. The human colon cancer cell lines (HT29, HCT116) were purchased from European Collection of Cell Cultures (ECACC). All cells were cultured and maintained in DMEM medium containing $10 \%$ foetal bovine serum and $1 \%$ penicillin and streptomycin at $37^{\circ} \mathrm{C}$ with $5 \% \mathrm{CO}_{2} / 95 \%$ air. Cells used in all experiments were at 3-8 passages after their receipt from the supplier.

Establishment of 5-FU resistance in colorectal cancer cells. HT29 and HCT116 human colorectal cancer cell lines were used to assess 5-FU resistance. Cells were seeded in 96-well plates with DMEM medium containing 10\% FBS and 1\% PenStrep at the density of $2 \times 10^{4}$ cells/well in $37^{\circ} \mathrm{C}$ with $5 \%$ $\mathrm{CO}_{2} / 95 \%$ air for $24 \mathrm{~h}$. After seeding, cells were washed three times with medium and then treated with 5 -FU for $48 \mathrm{~h}$. Following the treatment, cells were subjected to cell death and viability assays. In order to verify that 5 -FU resistance exists in these cancer cells, both cell lines were subjected to the treatment with a widen range of concentration of 5-FU for $48 \mathrm{~h}$. All experiments were performed in triplicate and repeated on two separately-initiated cultures $(n=6)$.

Determination of which MAPK pathways affected by 5-FU treatment. HT29 CRC cells were seeded in 6-well plates with DMEM medium containing 10\% FBS and 1\% PenStrep at the density of $1.8 \times 10^{5}$ cells/well in $37^{\circ} \mathrm{C}$ with $5 \% \mathrm{CO}_{2} / 95 \%$ air for $24 \mathrm{~h}$. Following the seeding, cells were treated by 5 -FU for $48 \mathrm{~h}$. The level of p38, phospho-p38, p42/44 and phosphorp42/44 was determined by Western blotting. The experiments were performed in duplicate and repeated on two separatelyinitiated cultures. The value of protein level in each treatment was expressed as the mean of 4 samples $(n=4)$.

Inhibition of p38 MAPK pathway. After establishment of 5-FU resistance, only 5-FU resistant cells (HT29) were used in the following experiments. Cells were seeded on 96-well plates with DMEM medium containing 10\% FBS and 1\% PenStrep at the density of $2 \times 10^{4}$ cells $/$ well in $37^{\circ} \mathrm{C}$ with $5 \%$ $\mathrm{CO}_{2} / 95 \%$ air for $24 \mathrm{~h}$. After seeding, cells were treated with or without 5-FU in combination with a p38 MAPK inhibitor (SB203580) or a MEK1/2 MAPK inhibitor (MEK1/2 inhibitor) for $48 \mathrm{~h}$. Following the treatment, cells were subjected to cell viability, death and caspase activity assays. The experiments were performed in triplicate and repeated on two separatelyinitiated cultures $(n=6)$.

Determination of the level of anti- and pro-apoptotic proteins following 5-FU treatment combined with 338 MAPK inhibition. HT29 CRC cells were seeded in 6-well plates with DMEM medium containing 10\% FBS and 1\% PenStrep at the density of $1.8 \times 10^{5}$ cells/well at $37^{\circ} \mathrm{C}$ with $5 \% \mathrm{CO}_{2} / 95 \%$ air for $24 \mathrm{~h}$. Following the seeding, cells were divided into 4 groups as follows, i) treated with 5 -FU $(5 \mu \mathrm{g} / \mathrm{ml})$, ii) received 5-FU $(5 \mu \mathrm{g} / \mathrm{ml})$ and SB203580 $(5 \mu \mathrm{M})$ treatment, iii) treated with 5-FU $(5 \mu \mathrm{g} / \mathrm{ml})$ and SB203580 $(10 \mu \mathrm{M})$, and iv) was untreated as the control group. The levels of Bax and $\mathrm{Bcl} 2$ were determined by Western blotting. The experiments were performed in duplicate and repeated on two separately-initiated cultures. The value of protein level in each treatment was expressed as the average of samples $(n=4)$.

Cell viability assay. Cell viability was assessed using a previously reported method (10). For comparison, all cell viability data were presented as the percentage of treated cells to untreated cells, which was calculated using the following formula: cell viability $(\%)=\mathrm{Ft} / \mathrm{Fc} \times 100$; where $\mathrm{Ft}$ represents fluorescence reading for treated cells and $\mathrm{Fc}$ represent fluorescence reading for untreated cells in the cell viability assay.

Cell death assay. Cell death assessment was carried out with a previously described method $(10,11)$. For comparison, the cell death index for treated and untreated cells was calculated with the formula: cell death index $=\mathrm{Ft} / \mathrm{Fc}$; where $\mathrm{Ft}$ and $\mathrm{Fc}$ represent the units of fluorescence (RLU) in the treated $(\mathrm{Ft})$ and the untreated $(\mathrm{Fc})$ cells, respectively.

Caspase activity assay. Caspase 8 and 9 activity was assessed with Caspase-Glo assay kit using a previously reported method (12). For comparison, all caspase activities were presented as increase fold-change compared to untreated cells.

Protein extraction and Western blotting. Cells were washed 3 times with PBS, lysed by the addition of RIPA buffer containing protease inhibitor cocktail (Roche) and harvested with a cell scraper. In order to assist cell lysis, cell suspensions were repeatedly frozen and thawed. SDS-PAGE was used to separate the proteins. The primary antibody dilution for Bax, Bcl2 was 1/200, for p38, phospho-p38, p42/44 and phosphor-p42/44 was $1 / 1000$. The density of protein bands was measured using densitometry software (Molecular Analyst, Bio-Rad image analysis software V1.5, USA).

Statistical analysis. All data have been examined and demonstrated a normal distribution. All results were expressed as mean \pm SEM. One way ANOVA (Prism version 42004 edition, USA) with multiple comparison tests were used. Statistical analysis was performed on samples $(n=4-6)$ and Bonferroni's multiple comparison test was used. $\mathrm{P}<0.05$ was considered statistically significant, $\mathrm{P}<0.01$ highly significant. In figures, indicated as ${ }^{*} \mathrm{P}<0.05$ and $^{* *} \mathrm{P}<0.01$, respectively. 


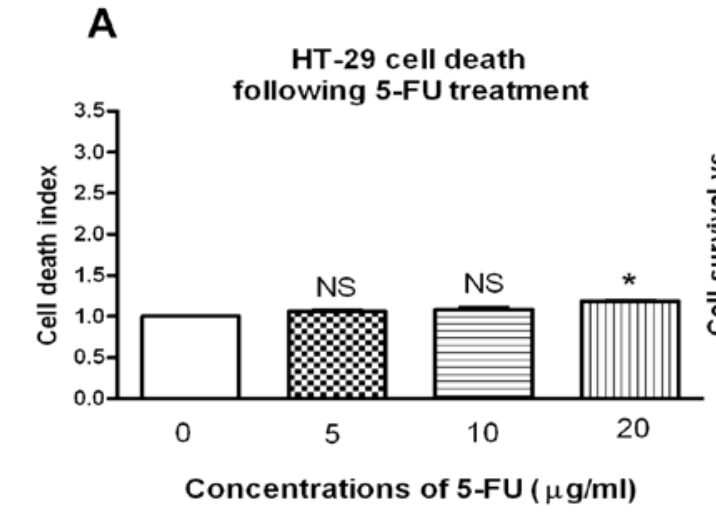

\section{B HT -29 cell survival}
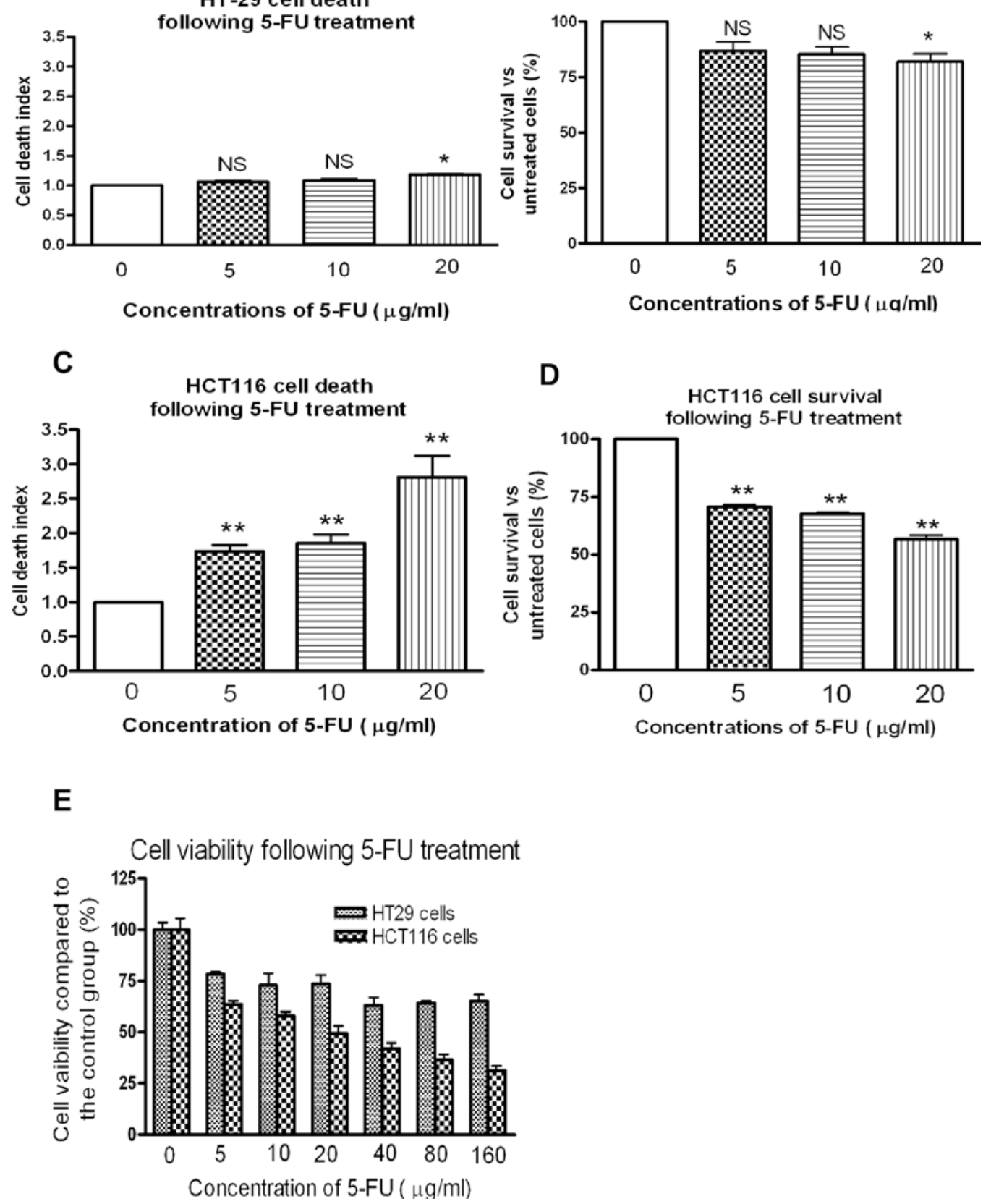

Figure 1. Human colon cancer HT29 (A and B) and HCT116 (C and D) cells were treated with 5-FU. Cell viabilities (B and D) and death index (A and C) were determined. The results are shown as mean \pm SEM, $(n=6)$. The viability of two cell lines were assessed following expose to a wide range of concentrations of 5-FU for $48 \mathrm{~h}$, the results (E) showed that when 5-FU concentration increased, the viability of HCT116 cell continuously decreased. The viability of HT29 cell only responded to the first few concentrations, but failed to change in response to the higher doses of 5-FU.

\section{Results}

Establishment 5-FU resistance in colorectal cancer cells. Two cell lines (HT29 and HCT116) were used to assess the resistance to 5-FU in CRC cells. Although 5-FU treatment was shown to induce cell death and reduce cell viability in both cell lines in a dose-dependent manner (Fig. 1), the sensitivity to the treatment was different in the two cell lines. HCT116 cells were more sensitive to the treatment. All doses of 5-FU induced significantly higher levels of cell death, and markedly reduced cell viabilities in HCT 116 cells (Fig. 1C and D). HT29 cells were much less sensitive to the treatment; only higher doses of 5-FU $(20 \mu \mathrm{g} / \mathrm{ml})$ induced higher levels of cell death and reduced cell viabilities significantly. Lower doses of 5-FU (5 and $10 \mu \mathrm{g} / \mathrm{ml}$ ) failed to kill HT29 cells (Fig. 1A and B). To further verify that 5-FU resistance exists in HT29 cells, two cell lines were treated with a wide range of concentrations of 5-FU, the result was shown in Fig. 1E. When 5-FU concentration increased, the viability of HCT116 cell continuously decreased. However the viability of HT29 cells only responded to the first few concentrations, but failed to response to the higher doses of 5-FU (Fig. 1E). This result established that HT29 cells are 5-FU resistant which also was confirmed by a recent study (13). Therefore, only the HT29 
A

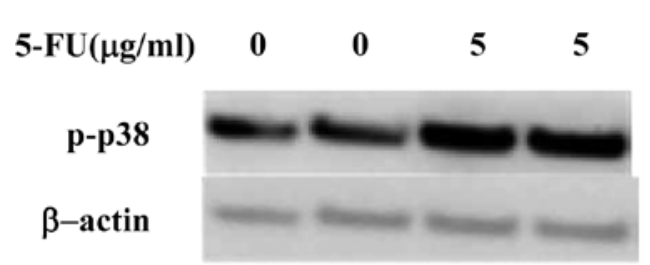

C

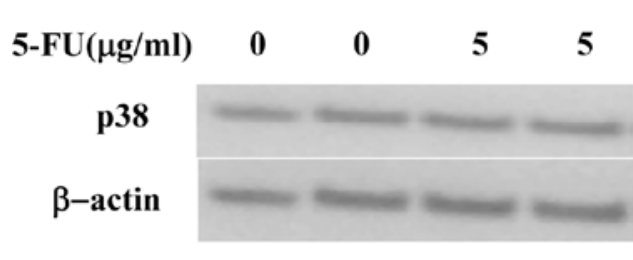

B $\quad$ p-p38 level

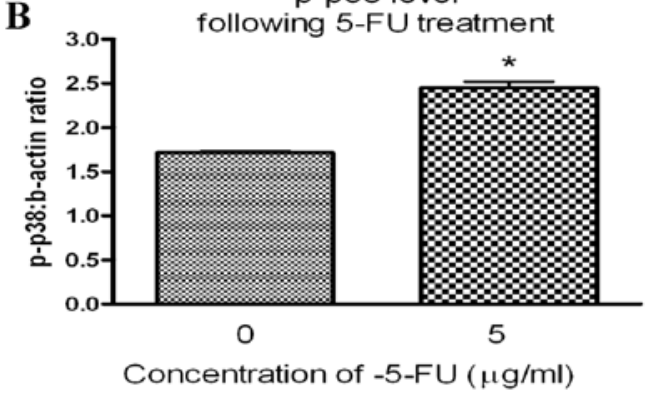

D

D38 level

following 5-FU treatment

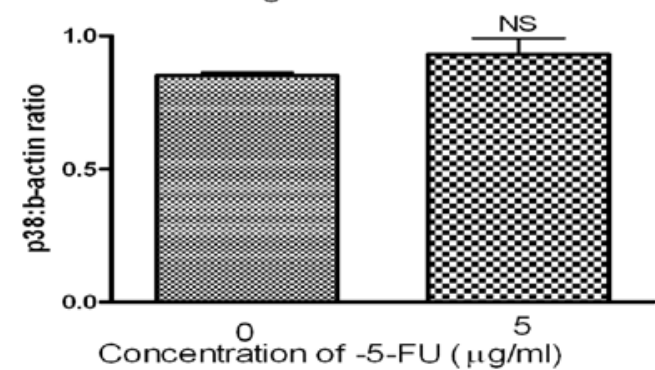

Figure 2. HT29 CRC cells were treated by 5-FU for $48 \mathrm{~h}$. Total protein was isolated from cells and separated with SDS-PAGE electrophoresis. The bands of phospho-p38 (A) and p38 (C) were identified by polyclonal antibodies against human phospho-p38 and p38, respectively. The level of phospho-p38 (B) and p38 (D) was determined by measurement of protein band using densitometry software. The results are shown as mean \pm SEM, $(n=4)$.

cells were used in the following investigation to sensitise cancer cells to 5-FU.

Determination of which MAPK pathway was affected by 5-FU treatment. The responses of two major signalling cascades (p38 and MEK1/2 MAPK pathways) to 5-FU were investigated. Results showed that the p38 MAPK pathway was affected by the treatment (Fig. 2). Following $48 \mathrm{~h}$ of 5-FU treatment, the levels of $\mathrm{p} 38$ protein were not significantly changed (Fig. 2C and D, P>0.05), but the level of phosphop38 was elevated significantly compared to the untreated cells (Fig. 2A and B, P<0.05). This suggested that 5-FU significantly increased p38 phosphorylation in HT29 cells. For the MEK MAPK cascade, it was found that both levels of p42/44 and phospho-p42/44 were not changed following the treatment, indicating that MEK MAPK pathway was not affected by 5-FU actions (Fig. 3).

Inhibition of p38 MAPK enhances 5-FU-induced cell death and reduces cell survival in CRC cells. In order to assess whether inhibition of p38 MAPK affected 5-FU treatment on HT29 cells, a p38 MAPK inhibitor (SB203580) was combined with 5-FU to treat HT29 cells. When cells were treated by 5-FU alone, there was no significant difference in cell death (Fig. 4A) and viability (Fig. 4B) compared to the untreated control cells, but if 5-FU was supplemented with SB203580, cell death significantly increased (Fig. 4A). Similar effects were also observed in cell viability assays (Fig. 4B). It is interesting to note that SB203580 enhanced 5-FU-induced cell death (Fig. 4A) and reduced cell viability (Fig. 4B) in a dose-dependent manner. All these data suggested that the inhibition of p38 MAPK pathway can sensitise HT29 cells to 5-FU.
Inhibition of MEK1/2 MAPK does not affect HT29 cancer cell sensitivity to 5-FU treatment. To investigate the role of MEK1/2 MAPK pathway in the development of 5-FU resistance in CRC cells, a MEK1/2 MAPK inhibitor (MEK1/2 inhibitor) was combined with 5-FU to treat HT29 cells. The results (Fig. 4C and D) showed that inhibition of MEK1/2 pathway did not affect the outcome of 5-FU treatment. There were no differences in cell death in any of the treated cells, including those treated by $5-\mathrm{FU}$ alone and 5-FU supplemented with MEK1/2 inhibitor compared to untreated cells (Fig. 4C). This indicated that MEK1/2 MAPK is not involved with cell apoptosis induced by 5 -FU. For cell viability, 5-FU treatment alone decreased the viable cell slightly, but the addition of MEK1/2 inhibitor failed to enhance 5-FU-reduced cell viability (Fig. 4D). All these data suggested that MEK1/2 MAPK pathway had no effect on 5-FU treatment in CRC cells, which is consistent with some previous studies $(14,15)$.

Inhibition of p38 MAPK significantly increases caspase 8 and 9 activity. Activation of caspases has been regarded as a hallmark for cell apoptosis. Further investigation was carried out to assess the effect of inhibition of p38 MAPK on the activity of caspases 8 and 9. Results show that the SB203580 combination with 5-FU was able to increase the activity of both caspase 8 and 9 significantly compared to untreated and 5-FU only treated cells (Fig. 5A and B). The SB203580 alone is also able to activate both caspases, but the combination appears to have more potential to stimulate both enzymes activities. Caspase 8 activation is one part of extrinsic apoptotic cascades and caspase 9 activation is one part of intrinsic apoptosis pathway. SB203580 treatment activated both caspase 8 and 9 indicating that interfering with p38 MAPK pathway induced CRC cell extrinsic and intrinsic apoptotic death. 
A

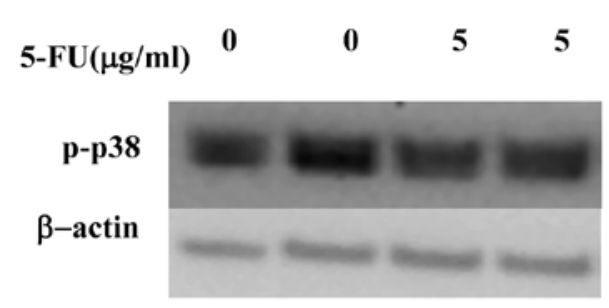

C

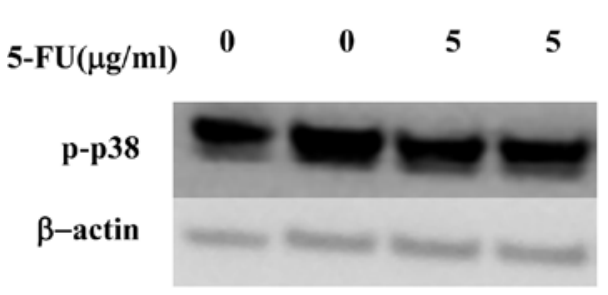

Level of p-p42/44

B following 5-FU treatment

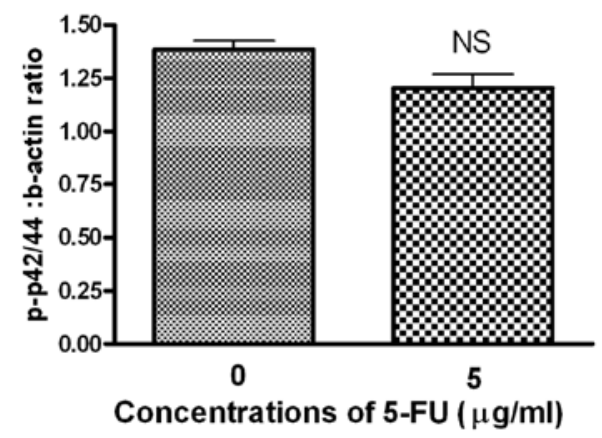

D

Level of p42/44 following 5-FU treatment

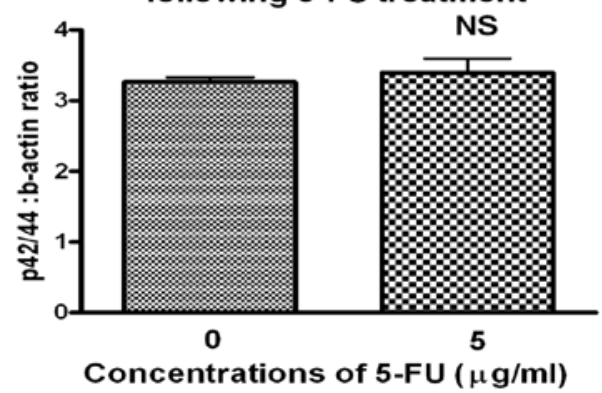

Figure 3. HT29 CRC cells were treated with 5-FU. Total protein was isolated from cells and separated with SDS-PAGE electrophoresis. The bands of phospho-p42/44 (A) and p42/44 (C) were identified by polyclonal antibodies against human phospho-p42/44 and p42/44, respectively. The levels of phosphop42/44 (B) and p42/44 (D) were determined by measurements of protein band density using densitometry software. The results are shown as mean \pm SEM, $(\mathrm{n}=4)$.

A

HT - 29 cells ( 48 hours)

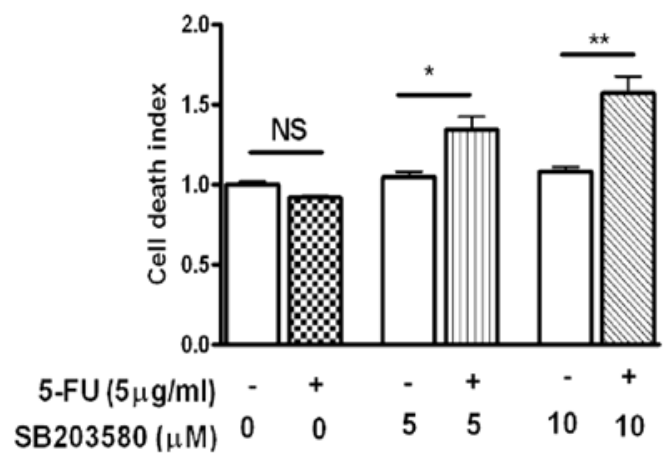

C

HT29 cell (48hours)

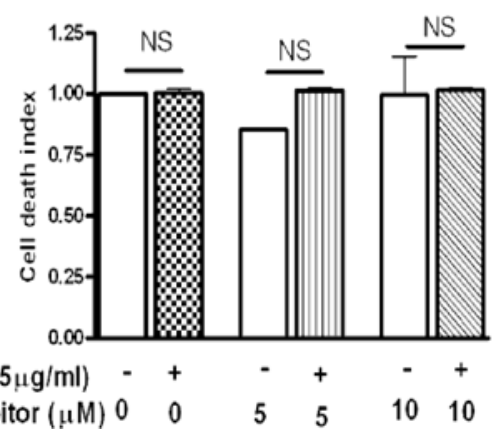

B

HT -29 cell (48 hours)

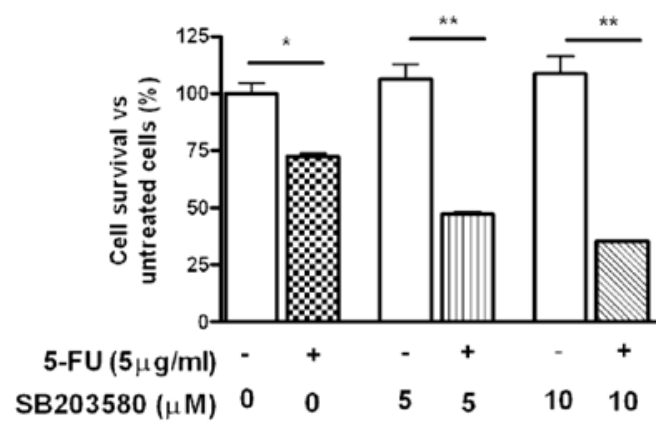

D

HT 29 cell ( 48 hours)

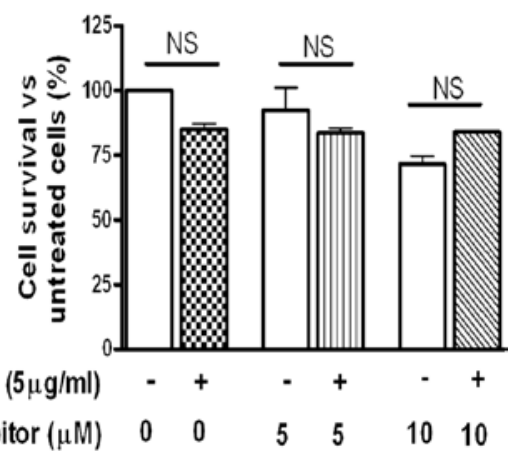

Figure 4. HT29 CRC cells were treated with 5-FU supplemented with or without SB203580 (A and B) or MEK1/2 inhibitor (C and D) inhibitor for 48 h. Cell death index $(A$ and $C)$ and viabilities $(B$ and $D)$ were determined by a previous reported method. The results are shown as mean \pm SEM, $(n=6)$. 
A

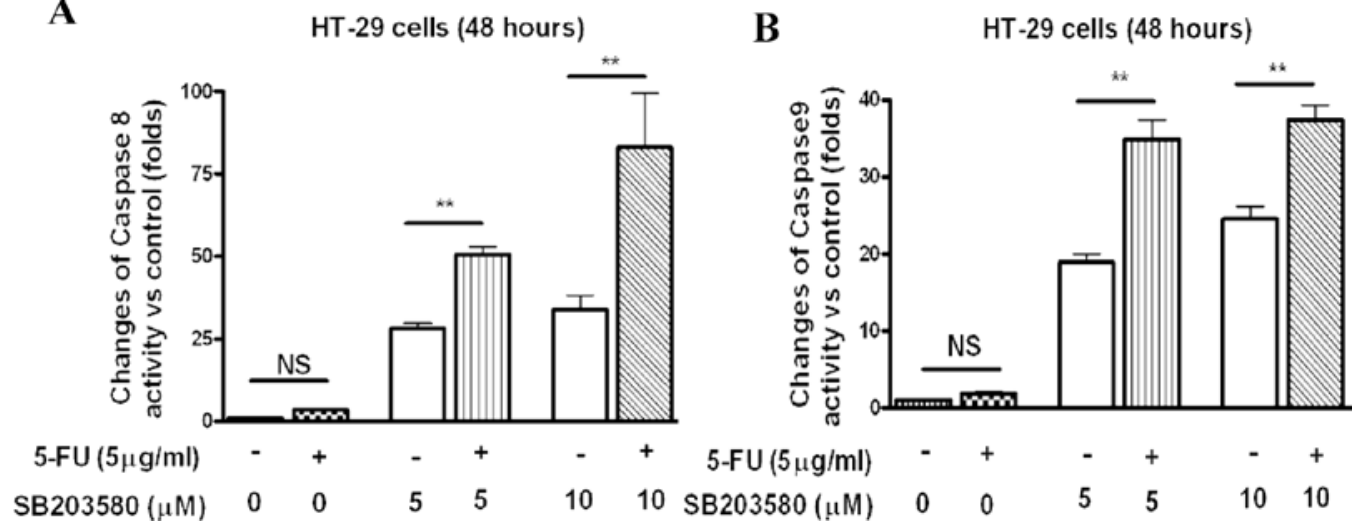

Figure 5. HT29 CRC cells were treated with 5-FU supplemented with or without SB203580 at concentrations of 5 and $10 \mu \mathrm{M}$ for $48 \mathrm{~h}$. The activities of caspase 8 (A) and 9 (B) were determined by Caspase-Glo assays. For comparisons, all caspase activities were presented as increase in fold compared with untreated cells. All results were expressed as means \pm SEM, $(n=6)$.

A

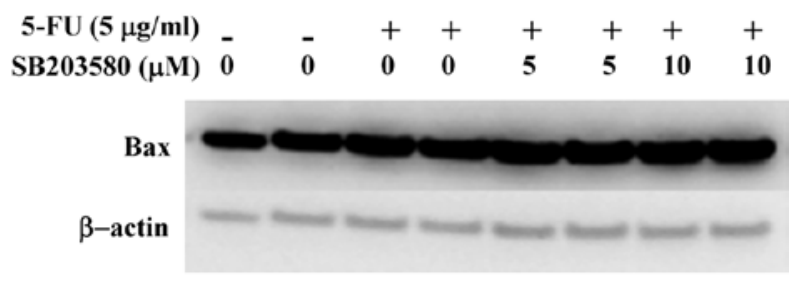

B

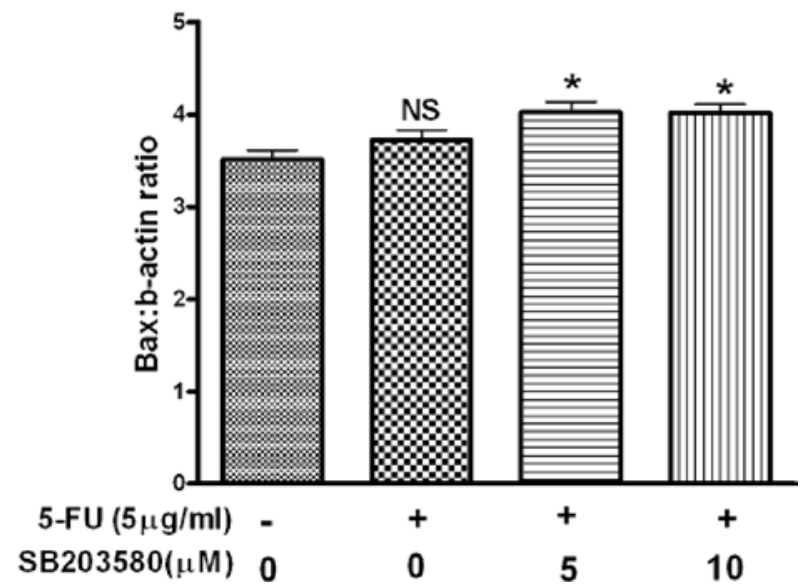

Figure 6. HT29 CRC cells were treated with 5-FU supplemented with SB203580. Total protein was isolated from cells and separated with SDS PAGE electrophoresis. The bands of $\operatorname{Bax}(\mathrm{A})$ and $\beta$-actin were identified by polyclonal antibodies against human Bax and $\beta$-actin. The level of BAX and $\beta$-actin was determined by measuring the density of protein bands using densitometry software and expressed as Bax: $\beta$-actin ratio (B). Results are shown as mean \pm SEM, $(n=4)$.

Inhibition of p38 MAPK increases pro-apoptotic protein Bax, but does not affect anti-apoptotic protein Bcl2. To test whether 5-FU supplement with p38MAPK have any effects on apoptotic regulation proteins, the expression levels of Bax (a pro-apoptotic protein) and Bcl2 (an anti-apoptotic protein) were examined by Western blotting following 5-FU alone or 5-FU supplemented with SB203580 treatment. The results
A

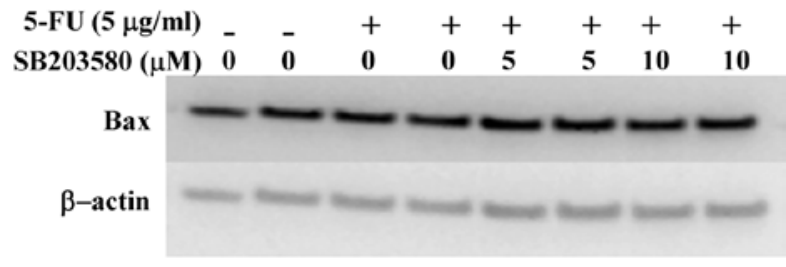

B

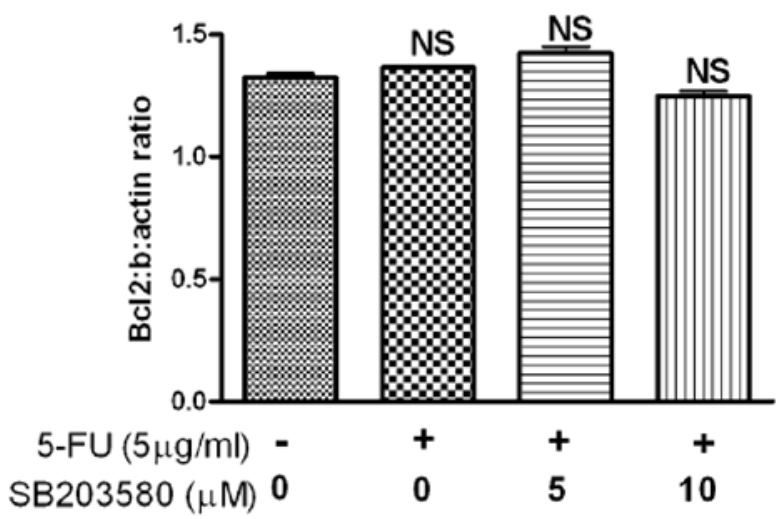

Figure 7. HT29 CRC cells were treated with 5-FU supplemented with SB203580. Total protein was isolated from cells and separated with SDSPAGE electrophoresis. The bands of $\mathrm{Bcl} 2$ (A) and $\beta$-actin were identified by polyclonal antibodies against human $\mathrm{Bcl} 2$ and $\beta$-actin, respectively. The levels of $\mathrm{Bcl} 2$ and $\beta$-actin were determined by measuring the density of protein bands using densitometry software and expressed as Bcl2: $\beta$-actin ratio $(\mathrm{B})$.

are shown in Figs. 6 and 7. It is shown that 5-FU alone was not able to alter expression of either Bax or Bcl2 (Figs. 6 and 7). When cells were treated with 5-FU supplemented with SB203580 (5-10 $\mu \mathrm{M})$, the level of pro-apoptotic protein Bax significantly increased (Fig. 6A and B). However, such effects were not observed in the anti-apoptotic protein Bcl2. These data suggested that 5-FU treatment supplemented with 
SB203580-induced HT29 cell death was via the increase in the expression of pro-apoptotic protein Bax.

\section{Discussion}

This study examined the effects of 5-FU on p38 MAPK and MEK1/2 pathways in a 5-FU-resistant CRC cell line, and found that 5-FU significantly increased p38 phosphorylation in CRC cells, which may give an advantage for cell survival from 5-FU treatment. When cancer cells were treated by 5-FU supplemented with p38 MAPK inhibitors, the advantage of survival disappeared and significant cell death was induced. The cell death appears to be correlated with increased expression of a pro-apoptotic protein, Bax. Further studies have confirmed that 5-FU supplemented with the inhibition of p38 MAPK significantly increased caspase 8 and 9 activity which is the hallmark of cellular apoptosis. We also found that 5-FU did not affect the MEK1/2 MAPK pathway, and inhibition of the MEK1/2 MAPK pathway did not enhance 5-FU killing in HT29 cells. All data together suggested that p38 MAPK is involved in cancer cell survival following 5-FU treatment, and the inhibition of P38 MAPK can enhance 5-FU killing CRC cells.

The mitogen-activation protein kinase (MAPK) pathway is a major signal transduction cascade which ultimately triggers multiple biological cell responses following growth factor treatment or stress stimulation (16). So far several subfamilies of MAPKs including p38 MAPK pathway (17) and extracellular signal-regulated kinase (MEK1/2) pathway (18) have been identified. P38 MAPK pathway is primarily activated in response to environmental stress and mainly involved in cell growth arrest and apoptosis (17). It can be stimulated by various stresses such as UV and $\gamma$ irradiation, osmotic stress, heat shock and some chemotherapeutic drugs (19). A recent study (20) found that inhibition of p38 MAPK downregulated a multidrug-resistance I gene, which was believed to be responsible for chemotherapy resistance in gastric cancer cells. However, to date we could not find any report providing insight into the details of the role of p38 MAPK pathway in 5-FU resistance in CRC cells. In this study we examined the effects of 5-FU treatment on p38 MAPK pathways and found that 5-FU increased p38 phosphorylation. Inhibition of p38 MAPK markedly enhanced 5-FU-induced CRC cell death, and significantly improved 5 -FU-reduced CRC cell viability and increased caspases 8 and 9 activities, which up-regulate apoptosis induced by 5-FU. The mechanism of p38 MAPK inhibition to sensitise cancer cells to the 5-FU may be due to the increases in the expression of a pro-apoptotic protein Bax.

The MEK $1 / 2$ pathway is mainly activated by mitogen and growth factors, and participates in cellular processes such as cell proliferation and differentiation (18). The role of MEK signalling in cancer cell chemoresistance remains contradictory. Some studies have found that activation of MEK pathway is associated with resistance to apoptosis-induced by chemotherapeutic drugs, and inhibition of the MEK signalling leads to increased sensitivity of cancer cells to chemotherapy (21-23), alternatively some studies have found that inhibitors of MEK1/2 MAPK blocked chemotherapy-induced apoptosis $(24,25)$, whilst other studies have demonstrated that modulation of MEK1/2 MAPK does not affect chemoresistance profiles in cancer cells $(14,15)$. We found that the inhibition of the MEK1/2 MAPK pathway has no effect on 5-FU treatment in CRC cells, which is consistent with some of these contradictory findings $(14,15)$. The role for MEK 1/2 MAPK pathway in determining cell survival or death is far from clear (23). The different effects observed suggest that the role of MEK1/2 pathway in chemoresistance is dependent on cell type and conditions.

Bax and Bcl-2 are two main proteins which mediate mitochondrial membrane permeability (26). Bax is a pro-apoptotic protein, which forms a trans-membrane pore across the outer mitochondrial membrane, leading to loss of membrane potential and enhanced apoptosis. Bcl-2 is an anti-apoptotic protein, which prevents the pore formation and cell death (27). The ratio of $\mathrm{Bcl} 2$ to Bax can be a marker to indicate the propensity of cells to undergo apoptosis (26). Our study shows that when cells were treated with 5-FU supplemented with an inhibitor of p38 MAPK the level of pro-apoptotic protein (Bax) significantly increased, but the level of anti-apoptotic protein (Bcl2) was not affected. This suggests that the inhibition of p38 MAPK induces Bax expression, which in turn, modulated the loss of mitochondrial membrane potential, finally contributed to cancer cell death.

A recent study (28) which analysed data from $>20,000$ CRC patients in 18 randomised trials has shown that 5-FU-based chemotherapy has improved overall survival in CRC patients, but it also identified that the early recurrence of the cancer was a great challenge to further improve the response rates. There are clear correlations between chemoresistance and cancer recurrence. 5-FU-induced cancer cell apoptosis involves many cellular signalling pathways. Investigation into the detail of these pathways will help us to understand why some tumour cells exposed to 5-FU often lose sensitivity. This study provides more details of the role of the p38 MAPK pathway in the development of 5-FU resistance in CRC cells, and could be used to optimise adjuvant agents to improve the response rate of chemotherapeutic agents in CRC patients.

\section{Acknowledgements}

S.Y.Y. and M.C.W. have received financial support from Wellcom VIP Award. S.Y.Y. has received financial support from Motor Neurone Disease Association-UK and I.O.C. World Anti Doping Agency.

\section{References}

1. Ferlay J, Autier P, Boniol M, Heanue M, Colombet M and Boyle P: Estimates of the cancer incidence and mortality in Europe in 2006. Ann Oncol 18: 581-592, 2007.

2. Jemal A, Siegel R, Ward E, Murray T, Xu JQ and Thun MJ: Cancer statistics, 2007. CA Cancer J Clin 57: 43-66, 2007.

3. American Cancer Society: Cancer facts and figures 2008. Am Cancer Soc, Atlanta, 2008.

4. Jones DV Jr, Winn RJ, Brown BW, Levy LB, Pugh RP, Wade JL 3rd, Gross HM, Pendergrass KB, Levin B and Abbruzzese JL: Randomized phase III study of 5-fluorouracil plus high dose folinic acid versus 5-fluorouracil plus folinic acid plus methyl-lomustine for patients with advanced colorectal cancer. Cancer 76: 1709-1714, 1995.

5. Szoke D, Gyorffy A, Surowiak P, Tulassay Z, Dietel M and Gyorffy B: Identification of consensus genes and key regulatory elements in 5-fluorouracil resistance in gastric and colon cancer. Onkologie 30: 421-426, 2007. 
6. Pinedo HM and Peters GF: Fluorouracil: biochemistry and pharmacology. J Clin Oncol 6: 1653-1664, 1988.

7. Mader RM, Muller M and Steger GG: Resistance to 5-fluorouracil. Gen Pharmacol 31: 661-666, 1998.

8. Xu R, Sato N, Yanai K, Akiyoshi T, Nagai S, Wada J, Koga K, Mibu R, Nakamura M and Katano M: Enhancement of paclitaxelinduced apoptosis by inhibition of mitogen-activated protein kinase pathway in colon cancer cells. Anticancer Res 29: 261-270, 2009

9. Kuno Y, Kondo K, Iwata H, Senga T, Akiyama S, Ito K, Takagi H and Hamaguchi M: Tumor-specific activation of mitogen-activated protein kinase in human colorectal and gastric carcinoma tissues. Jpn J Cancer Res 89: 903-909, 1998.

10. Yang SY, Bolvin C, Sales KM, Fuller B, Seifalian AM and Winslet MC: IGF-I activates caspases 3/7, 8 and 9 but does not induce cell death in colorectal cancer cells. BMC Cancer 9: 158, 2009.

11. Remacle-Bonnet M, Garrouste F, Baillat G, Andre F, Marvaldi J and Pommier G: Membrane rafts segregate pro- from anti-apoptotic insulin-like growth factor-I receptor signaling in colon carcinoma cells stimulated by members of the tumor necrosis factor superfamily. Am J Pathol 167: 761-773, 2005.

12. Yang SY, Sales KM, Fuller BJ, Seifalian AM and Winslet MC: Inducing apoptosis of human colon cancer cells by an IGF-I D domain analogue peptide. Mol Cancer 7: 17, 2008

13. Akhdar H, Loyer P, Rauch C, Corlu A, Guillouzo A and Morel F: Involvement of Nrf2 activation in resistance to 5-fluorouracil in human colon cancer HT-29 cells. Eur J Cancer 45: 2219-2227, 2009.

14. Lee JT Jr, Steelman LS and McCubrey JA: Modulation of Raf/ MEK/ERK kinase activity does not affect the chemoresistance profile of advanced prostate cancer cells. Int J Oncol 26: 1637-1644, 2005.

15. Okano J and Rustgi AK: Paclitaxel induces prolonged activation of the Ras/MEK/ERK pathway independently of activating the programmed cell death machinery. J Biol Chem 276: 19555-19564, 2001.

16. Fang JY and Richardson BC: The MAPK signalling pathways and colorectal cancer. Lancet Oncol 6: 322-327, 2005.

17. Mansouri A, Ridgway LD, Korapati AL, Zhang Q, Tian L, Wang Y, Siddik ZH, Mills GB and Claret FX: Sustained activation of JNK/ p38 MAPK pathways in response to cisplatin leads to Fas ligand induction and cell death in ovarian carcinoma cells. J Biol Chem 278: 19245-19256, 2003.

18. Troppmair J, Bruder JT, Munoz H, Lloyd PA, Kyriakis J, Banerjee P, Avruch J and Rapp UR: Mitogen-activated protein kinase/extracellular signal-regulated protein kinase activation by oncogenes, serum, and 12-O-tetradecanoylphorbol-13-acetate requires Raf and is necessary for transformation. J Biol Chem 269: 7030-7035, 1994
19. Chang L and Karin M: Mammalian MAP kinase signalling cascades. Nature 410: 37-40, 2001.

20. Guo X, Ma N, Wang J, Song J, Bu X, Cheng Y, Sun K, Xiong H, Jiang G, Zhang B, Wu M and Wei L: Increased p38-MAPK is responsible for chemotherapy resistance in human gastric cancer cells. BMC Cancer 8: 375, 2008.

21. Jazirehi AR, Vega MI, Chatterjee D, Goodglick L and Bonavida B: Inhibition of the Raf-MEK1/2-ERK1/2 signaling pathway, Bcl-xL down-regulation, and chemosensitization of non-Hodgkin's lymphoma B cells by Rituximab. Cancer Res 64: 7117-7126, 2004.

22. Zelivianski S, Spellman M, Kellerman M, Kakitelashvilli V, Zhou XW, Lugo E, Lee MS, Taylor R, Davis TL, Hauke R and Lin MF: ERK inhibitor PD98059 enhances docetaxel-induced apoptosis of androgen-independent human prostate cancer cells. Int J Cancer 107: 478-485, 2003.

23. Zhao Y, Shen S, Guo J, Chen H, Greenblatt DY, Kleeff J, Liao Q, Chen G, Friess H and Leung PS: Mitogen-activated protein kinases and chemoresistance in pancreatic cancer cells. J Surg Res 136: 325-335, 2006.

24. Kim M, Yan Y, Kortum RL, Stoeger SM, Sgagias MK, Lee K Lewis RE and Cowan KH: Expression of kinase suppressor of Ras1 enhances cisplatin-induced extracellular signal-regulated kinase activation and cisplatin sensitivity. Cancer Res 65: 3986-3992, 2005.

25. Wang X, Martindale JL and Holbrook NJ: Requirement for ERK activation in cisplatin-induced apoptosis. J Biol Chem 275: 39435-39443, 2000.

26. Durai R, Yang SY, Sales KM, Seifalian AM, Goldspink G and Winslet MC: Insulin-like growth factor binding protein- 4 gene therapy increases apoptosis by altering Bcl-2 and Bax proteins and decreases angiogenesis in colorectal cancer. Int J Oncol 30: 883-888, 2007.

27. Zamzami N, Brenner C, Marzo I, Susin SA and Kroemer G: Subcellular and submitochondrial mode of action of Bcl-2-like oncoproteins. Oncogene 16: 2265-2282, 1998.

28. Sargent D, Sobrero A, Grothey A, O'Connell MJ, Buyse M, Andre T, Zheng Y, Green E, Labianca R, O'Callaghan C, Seitz JF, Francini G, Haller D, Yothers G, Goldberg R and de Gramont A: Evidence for cure by adjuvant therapy in colon cancer: observations based on individual patient data from 20,898 patients on 18 randomized trials. J Clin Oncol 27: 872-877, 2009. 